\title{
ANOTHER GRADED ALGEBRA WITH A NONRATIONAL HILBERT SERIES
}

\author{
YUJI KOBAYASHI
}

\begin{abstract}
Recently J. B. Shearer has constructed a graded algebra with a nonrational Hilbert series, a counterexample to Govorov's conjecture. In this note we give a simpler example.
\end{abstract}

Let $k\left\langle X_{1}, \ldots, X_{r}\right\rangle$ denote the free associative algebra in $r$ variables $X_{1}, \ldots, X_{r}$ of degree 1 over a field $k$. Let $I$ be a finitely-generated homogeneous ideal of $k\left\langle X_{1}, \ldots, X_{r}\right\rangle$. The quotient ring $R=k\left\langle X_{1}, \ldots, X_{r}\right\rangle / I$ is naturally a graded algebra over $k$. The Hilbert series of $R$ is the formal power series

$$
H_{R}(Z)=\sum_{n>0}\left(\operatorname{dim}_{k} R_{n}\right) Z^{n}
$$

where $R_{n}$ is the homogeneous component of $R$ of degree $n$.

V. E. Govorov conjectured that $H_{R}(Z)$ is a rational function [2], [3]. This conjecture has been of interest in connection with the problem of the rationality of the Poincaré series of local rings (see for example [4], [5]). Recently, J. B. Shearer [6] has given a counterexample to the conjecture. He has constructed a monoid algebra with a nonrational Hilbert series, which is generated by 10 elements with 57 quadratic relations. In this note we give a simpler example, a monoid algebra with a nonrational Hilbert series generated by 6 elements with 9 quadratic relations.

Let $F$ be a free monoid on a set $\left\{a, a^{+}, b, b^{+}, x, y\right\}$, and let $F^{0}=F \cup\{0\}$ be the monoid $F$ with a zero adjoined. We define a monoid $M$ as a quotient of $F^{0}$ modulo the following relations (i)-(iii).

(i) $a a^{+}=a^{+} a, b b^{+}=b^{+} b, a b^{+}=a^{+} b, b a^{+}=b^{+} a$.

(ii) $a x=x a, b x=x b^{+}, a^{+} y=y a^{+}, b y=y b^{+}$.

(iii) $x y=0$.

Two words $\rho$ and $\sigma$ in $F^{0}$ are called equivalent, if they are transformed to each other by a finite series of substitutions (i)-(iii). Let $M_{n}$ be the set of all nonzero classes of equivalent words of length $n$. Then

$$
M=\bigcup_{n>0} M_{n} \cup\{0\} \quad \text { (disjoint union). }
$$

For a subset $N$ of $M$, we define the formal power series $H_{N}(Z)$ called the Hilbert series of $N$ as

$$
H_{N}(Z)=\sum_{n>0}\left|N \cap M_{n}\right| Z^{n}
$$

Received by the editors March 14, 1979 and, in revised form, February 4, 1980.

AMS (MOS) subject classifications (1970). Primary 16A06, 20 M05. 
Let $R=k[M]$ be the monoid algebra of $M$ over $k$. Then we have

$$
H_{R}(Z)=H_{M}(Z)=\sum_{n>0}\left|M_{n}\right| Z^{n} .
$$

We will prove that $H_{M}(Z)$ is not rational.

We consider the following 8 subsets of $M$.

$A$ : the submonoid of $M$ generated by $a, a^{+}, b, b^{+}$.

$B$ (resp. $B^{+}$): the submonoid of $M$ generated by $a, b$ (resp. $a^{+}, b^{+}$),

$X$ (resp. $X^{*}$ ): the set of all equivalence classes of words in $a^{+}, b^{+}, x$ without $a^{+}$, $b^{+}$in the last (resp. first) position,

$Y$ (resp. $Y^{*}$ ): the set of all equivalence classes of words in $a, b, y$ without $a, b$ in the first (resp. last) position,

$C$ : the set of all equivalence classes of words in the form of $x \sigma y(\sigma \in A)$.

Easily we find

$$
\begin{aligned}
H_{A}(Z) & =1 /(1-2 Z)^{2}, \quad H_{B}(Z)=H_{B^{+}}(Z)=1 /(1-2 Z), \\
H_{X}(Z) & =H_{X^{*}}(Z)=H_{Y}(Z)=H_{Y^{*}}(Z) \\
& =1+Z /(1-3 Z)=(1-2 Z) /(1-3 Z) .
\end{aligned}
$$

Let $\rho$ be any word. By transferring every $x$ contained in $\rho$ to the left and $y$ to the right as much as possible applying substitutions (i) and (ii), we get a word $\bar{\rho}$ equivalent to $\rho$ whose every subword without $x, y$ appearing just before $x$ (resp. after $y$ ) consists of only $a^{+}, b^{+}$(resp. $a, b$ ). This $\bar{\rho}$ takes the form of

$$
\beta \phi_{1} \gamma_{1} \psi_{1} \phi_{2} \gamma_{2} \psi_{2} \ldots \phi_{s} \gamma_{s} \psi_{s} \beta^{\prime}
$$

where $\phi_{i} \in X^{*}, \psi_{i} \in Y^{*}, \gamma_{i} \in C$ for $i=1, \ldots, s$, and $\beta \in B^{+}$or $\beta=\sigma \psi y$ ( $\sigma \in$ $A, \psi \in Y$ ) and $\beta^{\prime} \in B$ or $\beta^{\prime}=x \phi \sigma(\sigma \in A, \phi \in X)$ ( $s$ is possibly 0 , that is, the subword between $\beta$ and $\beta^{\prime}$ could be empty). Conversely, to every expression given as (1) a unique equivalence class of words corresponds. Therefore the Hilbert series $H_{M}(Z)$ is given as

$$
\begin{aligned}
H_{M}(Z) & =\frac{\left(H_{B^{+}}(Z)+Z H_{A}(Z) H_{Y}(Z)\right)\left(H_{B}(Z)+Z H_{A}(Z) H_{X}(Z)\right)}{1-H_{X^{*}}(Z) H_{C}(Z) H_{Y^{*}}(Z)} \\
& =\frac{1}{(1-3 Z)^{2}-(1-2 Z)^{2} H_{C}(Z)}
\end{aligned}
$$

Thus the proof of the irrationality of $H_{M}(Z)$ is reduced to that of $H_{C}(Z)$.

Define $d(n)=\mid\left\{\sigma \in A \cap M_{n} \mid x \sigma y=0\right.$ in $\left.M\right\} \mid$ for $n>0$ and $D(Z)=$ $\Sigma_{n>0} d(n) Z^{n}$. Then we have $H_{C}(Z)=Z^{2}\left(H_{A}(Z)-D(Z)\right)$. We will show that $D(Z)$ is not rational.

Let $\sigma \in A$. Moving the $y$ in the last position of $x \sigma y$ leftward as much as possible using substitutions (i) and (ii), we get a word $x \sigma_{1} y \sigma_{2}$ equivalent to $x \sigma y$ such that $\sigma_{2} \in B^{+}$and $\sigma_{1}=1$ or

$$
\sigma_{1}=\tau_{1} a \quad\left(\tau_{1} \in B\right)
$$

or

$$
\sigma_{1}=\tau_{1} b^{+} \quad\left(\tau_{1} \in B^{+}\right)
$$


If $\sigma_{1}=1$, then $x \sigma y=0$. Let us consider case (2) (the argument on case (3) is parallel). For a word $\sigma \in A, \alpha(\sigma)$ (resp. $\beta(\sigma))$ denotes the number of $a$ 's (resp. $b$ 's) contained in the string of $a$ and $b$ obtained by dropping all +'s of $\sigma$. If the word $\sigma_{2}$ has a left divisor $\sigma_{3} \in B^{+}\left(\sigma_{2}=\sigma_{3} \sigma_{4}\right.$ for some $\left.\sigma_{4} \in B^{+}\right)$such that $\alpha\left(\sigma_{3}\right)-\beta\left(\sigma_{3}\right)$ $=\beta\left(\sigma_{1}\right)$, then

$$
x \sigma y=x \sigma_{1} y \sigma_{2}=x \sigma_{1} \bar{\sigma}_{3} y \sigma_{4}=\sigma_{1} \tilde{\sigma}_{3} x y \sigma_{4}=0,
$$

where $\bar{\sigma}_{3}$ (resp. $\tilde{\sigma}_{3}$ ) is the word obtained from $\sigma_{3}$ by changing every $b^{+}$in $\sigma_{3}$ to $b$ (resp. by dropping all + 's in $\sigma_{3}$ ). Conversely, $x \sigma y=0$ happens only under this situation.

Let $d(n, r)$ for $n, r \geqslant 0$ be the number of words $\sigma \in B$ of length $n$ having left divisors $\sigma^{\prime} \in B$ such that $\alpha\left(\sigma^{\prime}\right)-\beta\left(\sigma^{\prime}\right)=r$. Then the preceding argument gives

$$
d(n)=2^{n}+2 \cdot \sum_{r>0} \sum_{l+m=n-1}\left(\begin{array}{l}
l \\
r
\end{array}\right) d(m, r) .
$$

Let us define $D_{r}(Z)=\Sigma_{n>0} d(n, r) Z^{n}$. Using the formula

$$
\sum_{n>0}\left(\begin{array}{l}
n \\
r
\end{array}\right) Z^{n}=\frac{Z^{r}}{(1-Z)^{r+1}}
$$

we obtain from (4) that

$$
D(Z)=\frac{1}{1-2 Z}+\frac{2 Z}{1-Z} \cdot \sum_{r>0}\left(\frac{Z}{1-Z}\right)^{r} D_{r}(Z) .
$$

Clearly we have

$$
d(n, 0)=2^{n} \text { and } d(n, r)=0 \text { for } n<r
$$

In particular

$$
D_{0}(Z)=1 /(1-2 Z)
$$

For $n, r>1$ we see

$$
d(n, r)=d(n-1, r-1)+d(n-1, r+1) .
$$

It follows from (6) and (8) that

$$
D_{r}(Z)=Z\left(D_{r-1}(Z)+D_{r+1}(Z)\right)
$$

for $r \geqslant 1$. Let $e(n)$ be the number of sequences $\varepsilon_{1}, \ldots, \varepsilon_{n}$ consisting of 1 and -1 of length $n$ such that $1+\varepsilon_{1}+\cdots+\varepsilon_{n}=0$ and $\varepsilon_{1}+\cdots+\varepsilon_{i}>0$ for all $i<n$. We define $e(0)=0$ for convenience. Now define

$$
E(Z)=\sum_{n>0} e(n) Z^{n}
$$

From (9) we obtain

$$
D_{1}(Z)=D_{0}(Z) E(Z) \text {. }
$$

Clearly $e(1)=1$. From the definition of $e(n)$ we see

$$
e(n)=\sum_{l+m=n-1} e(l) e(m)
$$

for $n \geq 2$. Therefore we have

$$
E(Z)=Z+Z(E(Z))^{2}
$$


This means $E(Z)$ is not rational (but algebraic). (11) also means that $E(Z)$ satisfies the characteristic equation $Z X^{2}-X+Z=0$ of the linear difference equation (9). Hence observing (7) and (10) we get

$$
D_{r}(Z)=D_{0}(Z)(E(Z))^{r}=(E(Z))^{r} /(1-2 Z) .
$$

Therefore by (5) we obtain

$$
\begin{aligned}
D(Z) & =\frac{1}{1-2 Z}+\frac{2 Z}{(1-2 Z)(1-Z)} \cdot \sum_{r>0}\left(\frac{Z E(Z)}{1-Z}\right)^{r} \\
& =\frac{1}{1-2 Z}\left(1+\frac{2 Z}{1-Z-Z E(Z)}\right) .
\end{aligned}
$$

This shows that $D(Z)$ is not rational. Consequently $H_{M}(Z)$ is not rational either.

REMARK 1. The number of the relations cannot be reduced to 1 , because a single relation always gives rise to a rational Hilbert series by Backelin [1].

REMARK 2. Recently Ufnarovsky [7] has shown that a graded algebra with a nonrational Hilbert series is constructed as a universal enveloping algebra of a certain Lie algebra. But there a generator of degree greater than 1 is needed. See also [6, Added in proof].

ACKNOWLEDGMENT. The author wishes to thank the referee for valuable suggestions without which he could not have given the example in the present form.

\section{REFERENCES}

1. J. Backelin, La série de Poincaré-Betti d'une algèbre graduée de type fini à une relation est rationnelle, C. R. Acad. Sci. Paris Ser. A 287 (1978), 843-846.

2. V. E. Govorov, Graded algebras, Math. Notes 12 (1972), 552-556. (Transl. of Mat. Zametki 12 (1972), 197-204).

3. $\ldots$, On the dimension of graded algebras, Math. Notes 14 (1973), 678-682. (Transl. of Mat. Zametki 14 (1973), 209-216.)

4. Y. Kobayashi, The Hilbert series of some graded algebras and the Poincare series of some local rings, Math. Scand. 42 (1978), 19-33.

5. J. E. Roos, Relations between the Poincaré-Betti series of loop spaces and of local rings, Lecture Notes in Math., Vol. 740, Springer-Verlag, Berlin and New York, 1979, pp. 285-322.

6. J. B. Shearer, A graded algebra with a non-rational Hilbert series, J. Algebra 62 (1980), 228-231.

7. V. A. Ufnarovsky, On algebras' growth, Vestnik Moskov. Univ. Ser. I Mat. Meh. 1978, no. 4, 59-65. (Russian)

Faculty of Education, Tokushma University, Mnami-Josanjma, Tokushma, Japan 